IJMMS 32:10 (2002) 579-585

PII. S0161171202204196

http://ijmms.hindawi.com

(c) Hindawi Publishing Corp.

\title{
AN ENTROPIC REGULARIZATION METHOD FOR SOLVING SYSTEMS OF FUZZY LINEAR INEQUALITIES
}

\author{
F. B. LIU
}

Received 23 April 2002

\begin{abstract}
Solving systems of fuzzy linear inequalities could lead to the solutions of fuzzy linear programs. It is shown that a system of fuzzy linear inequalities can be converted to a regular min-max problem. An entropic regularization method is introduced for solving such a problem. Some computational results are included.
\end{abstract}

2000 Mathematics Subject Classification: 90C70, 65K05, 65K10.

1. Introduction. Over the past years, the field of fuzzy linear programming has experienced a great deal of growth [10]. In contrast to classical linear programming problems, fuzzy linear programming problems do not have one unique model. Many variations are possible depending on the assumptions or features of the real situation to be modeled. Various models and approaches have been devised in the literature to deal with the diversity in fuzzy linear programming problems. In this work, we consider solving a fuzzy linear programming problem in view of the system of fuzzy linear inequalities

$$
f_{i}(\mathbf{x}) \lesssim 0, \quad i=1,2, \ldots, m,
$$

where $f_{i}(\mathbf{x})$ are linear real-valued functions in $\mathbf{x} \in \mathbb{R}^{n}$ and "ミ” denotes the fuzzified version of " $\leq$ " with the linguistic interpretation "approximately less than or equal to." Each fuzzy inequality $f_{i}(\mathbf{x}) \leqq 0$ actually determines a fuzzy set $\tilde{C}_{i}$, whose membership function is denoted by $\mu_{C_{i}}$. The membership grade $\mu_{C_{i}}(\mathbf{x})$ can be interpreted as the degree to which the regular inequality $f_{i}(\mathbf{x}) \leq 0, i=1,2, \ldots, m$, is satisfied. To specify the membership functions $\mu_{C_{i}}$, it is commonly assumed that $\mu_{C_{i}}(\mathbf{x})$ should be 0 if the regular linear inequality $f_{i}(\mathbf{x}) \leq 0$ is strongly violated and 1 if it is satisfied. This leads to a membership function in the form

$$
\mu_{C_{i}}(\mathbf{x})= \begin{cases}1, & \text { if } f_{i}(\mathbf{x}) \leq 0, \\ \mu_{i}\left(f_{i}(\mathbf{x})\right), & \text { if } 0<f_{i}(\mathbf{x}) \leq t_{i}, \\ 0, & \text { if } f_{i}(\mathbf{x})>t_{i},\end{cases}
$$

where $i=1,2, \ldots, m$ and $t_{i} \geq 0$ is the tolerance level which a decision maker can tolerate in the accomplishment of the fuzzy inequality $f_{i}(\mathbf{x}) \leqq 0$. We usually assume that $\mu_{i}\left(f_{i}(\mathbf{x})\right) \in[0,1]$ and it is continuous and strictly decreasing over $\left[0, t_{i}\right]$. Figure 1.1 shows different shapes of such membership functions. 


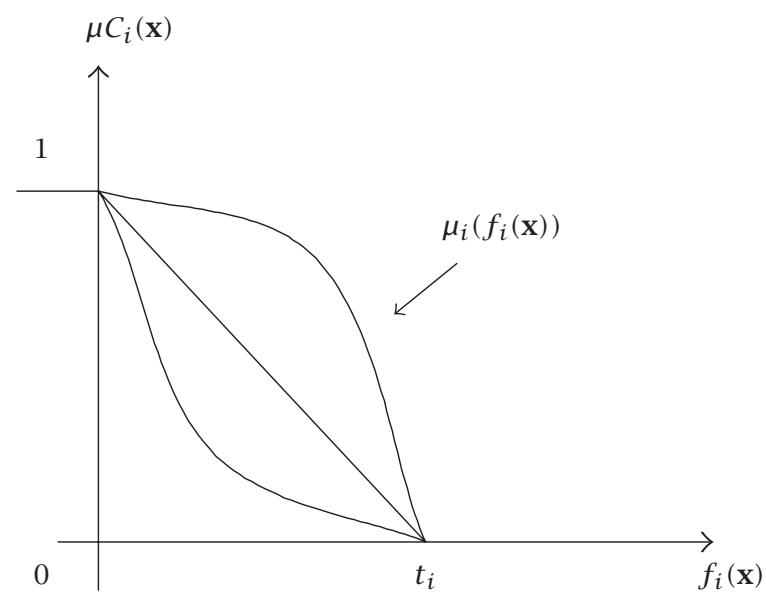

FIGURE 1.1. The membership function $\mu_{C_{i}}(\mathbf{x})$ of the fuzzy inequality $f_{i}(\mathbf{x}) \lesssim 0$.

Let a fuzzy decision $\tilde{D}$ of system (1.1) be defined as the fuzzy set resulting from the intersection of $\tilde{C}_{i}, i=1,2, \ldots, m$, with a corresponding membership function

$$
\mu_{\tilde{D}}(\mathbf{x})=\min _{i=1,2, \ldots, m}\left\{\mu_{C_{i}}(\mathbf{x})\right\}
$$

According to $[1,17]$, a solution, say $\mathbf{x}^{*}$, of the system of fuzzy linear inequalities (1.1) can be taken as the solution with the highest membership in the fuzzy decision set $\tilde{D}$ and obtained by solving the problem

$$
\max _{\mathbf{x} \in \mathbb{R}^{n}} \min _{i=1,2, \ldots, m}\left\{\mu_{C_{i}}(\mathbf{x})\right\}
$$

which is equivalent to the min-max problem

$$
-\min _{\mathbf{x} \in \mathbb{R}^{n}} \max _{i=1,2, \ldots, m}\left\{-\mu_{C_{i}}(\mathbf{x})\right\}
$$

It is well known that the nondifferentiability of the max function

$$
F(\mathbf{x}) \triangleq \max _{i=1,2, \ldots, m}\left\{\mu_{C_{i}}(\mathbf{x})\right\}
$$

presents difficulty in finding an optimal solution. In this work, a regularization method using entropy functions is introduced to derive a smooth and good approximation function in explicit expression for the max function. The organization of the rest of this paper is as follows. Section 2 describes the entropic regularization method and shows that a system of fuzzy linear inequalities with concave membership function can be converted to a regular unconstrained convex programming problem. A numerical example is provided in Section 3 to illustrate the solution procedure. Section 4 concludes the paper by making some remarks. 
2. Entropic regularization method. To find a solution of the system of fuzzy linear inequalities (1.1), we consider problem (1.5). One major difficulty encountered in developing solution methods for the min-max problem (1.5) is the nondifferentiability of the max function

$$
F(\mathbf{x}) \triangleq \max _{i=1,2, \ldots, m}\left\{-\mu_{C_{i}}(\mathbf{x})\right\}
$$

A distinct feature of the recent development centers around the idea of developing "smooth algorithms" $[4,5,13,14,15,16]$. Among them, a class called "regularization methods" has been developed based on approximating the max function $F(\mathbf{x})$ by certain smooth functions $[2,3,5,14,16]$.

Note that the min-max problem (1.5) has the Lagrange function

$$
L(\mathbf{x}, \lambda) \triangleq \sum_{i=1}^{m} \lambda_{i}\left(-\mu_{C_{i}}(\mathbf{x})\right)
$$

for each $\mathbf{x} \in \mathbb{R}^{n}$, where the $m$-vector $\lambda$ denotes Lagrange multipliers which satisfy the nonnegativity and normality conditions, that is, these multipliers are restricted to fall within the simplex

$$
\Lambda \triangleq\left\{\lambda \mid \lambda \geq 0 ; \sum_{i=1}^{m} \lambda_{i}=1\right\} .
$$

It is obvious that the inequality

$$
L(\mathbf{x}, \lambda)=\sum_{i=1}^{m} \lambda_{i}\left(-\mu_{C_{i}}(\mathbf{x})\right) \leq \max _{i=1,2, \ldots, m}\left\{-\mu_{C_{i}}(\mathbf{x})\right\}=F(\mathbf{x})
$$

holds for any $\lambda \in \Lambda$. Under some regular assumptions, the max function $F(\mathbf{x})$ can be obtained as

$$
F(\mathbf{x})=\max _{\lambda \in \Lambda} L(\mathbf{x}, \lambda)
$$

Unfortunately, the maximization of $\sum_{i=1}^{m} \lambda_{i}\left(-\mu_{C_{i}}(\mathbf{x})\right)$ over $\lambda \in \Lambda$ rarely has an explicit solution $\lambda(\mathbf{x})$ for all $\mathbf{x} \in \mathbb{R}^{n}$. Therefore, general regularization methods consider the substitute (of $L(\mathbf{x}, \lambda)$ )

$$
L_{p}(\mathbf{x}, \lambda) \triangleq \sum_{i=1}^{m} \lambda_{i}\left(-\mu_{C_{i}}(\mathbf{x})\right)+\frac{1}{p} R(\lambda ; \beta),
$$

where $p>0$ is a control parameter, $R$ is a regularization function, and $\beta$ is an optional parameter vector of $R$. Carefully choosing the function $R$, maximizing the substitute (2.6) could result in a smooth approximation function

$$
F_{p}(\mathbf{x}) \triangleq \max _{\lambda \in \Lambda} L_{p}(\mathbf{x}, \lambda)
$$


Some known regularization methods have considered certain regularization functions [5, 6, 7]; however, no explicit expression of $F_{p}(\mathbf{x})$ was given in these methods. Recently, a regularization method using entropy functions [11] is introduced to derive a smooth and good approximation function $F_{p}(\mathbf{x})$ in explicit expression for the max function $F(\mathbf{x})$. Given $\mathbf{x} \in \mathbb{R}^{n}$, when the Shannon's entropy function [8] is chosen as the regularization function, that is, $R(\lambda)=-\sum_{i=1}^{m} \lambda_{i} \ln \lambda_{i}$, we define

$$
L_{p}(\mathbf{x}, \lambda) \triangleq \sum_{i=1}^{m} \lambda_{i}\left(-\mu_{C_{i}}(\mathbf{x})\right)-\frac{1}{p} \sum_{i=1}^{m} \lambda_{i} \ln \lambda_{i},
$$

where $\lambda \in \Lambda$. Since $L_{p}(\mathbf{x}, \lambda)$ is strictly concave in $\lambda$, maximizing it over $\Lambda$ gives a unique optimal solution

$$
\lambda_{i}^{*}(\mathbf{x}, p)=\frac{\exp \left[p\left(-\mu_{C_{i}}(\mathbf{x})\right)\right]}{Z}, \quad i=1,2, \ldots, m
$$

where

$$
Z=\sum_{i=1}^{m} \exp \left[p\left(-\mu_{C_{i}}(\mathbf{x})\right)\right]
$$

Substituting $\lambda_{i}$ in (2.8) by $\lambda_{i}^{*}(\mathbf{x}, p)$ for (2.7) results in an explicit expression

$$
F_{p}(\mathbf{x})=\frac{1}{p} \ln \left\{\sum_{i=1}^{m} \exp \left[p\left(-\mu_{C_{i}}(\mathbf{x})\right)\right]\right\},
$$

which is a smooth function. It should be emphasized that the $F_{p}(\mathbf{x})$ function obtained here is indeed the maximum of $L_{p}(\mathbf{x}, \lambda)$ over $\Lambda$ for each $\mathbf{x} \in \mathbb{R}^{n}$. This procedure guarantees that, for any arbitrarily small $\epsilon>0$, an $\epsilon$-optimal solution of the min-max problem (1.5) can be obtained by solving the unconstrained smooth optimization problem

$$
\min _{\mathbf{x} \in \mathbb{R}^{n}} F_{p}(\mathbf{x})=\frac{1}{p} \ln \left\{\sum_{i=1}^{m} \exp \left[p\left(-\mu_{C_{i}}(\mathbf{x})\right)\right]\right\}
$$

with a sufficiently large $p$. It should be noted that, in practice, an accurate approximation can be obtained using a moderately large $p$. Also, because of the special "logexponential" form of $F_{p}(\mathbf{x})$, it is highly smooth and avoids most overflow problems in computation. Moreover, since it is an unconstrained, smooth optimization problem, the commonly used solution methods, such as the BFGS subroutines [12], can be readily applied.

LEMMA 2.1. If $\mu_{C_{i}}(\mathbf{x}), i=1,2, \ldots, m$, are concave over a convex set $\Omega$ in $\mathbb{R}^{n}$, then $F_{p}(\mathbf{x})$ is convex. 
Proof. For any $\mathbf{x}_{1}, \mathbf{x}_{2} \in \mathbb{R}^{n}$ and $\lambda \in(0,1)$, we have

$$
\begin{aligned}
F_{p}\left(\lambda \mathbf{x}_{1}+(1-\lambda) \mathbf{x}_{2}\right) & =\frac{1}{p} \ln \sum_{i=1}^{m} \exp \left[p\left(-\mu_{C_{i}}\left(\lambda \mathbf{x}_{1}+(1-\lambda) \mathbf{x}_{2}\right)\right)\right] \\
& \leq \frac{1}{p} \ln \sum_{i=1}^{m} \exp \left[\lambda p\left(-\mu_{C_{i}}\left(\mathbf{x}_{1}\right)\right)+(1-\lambda) p\left(-\mu_{C_{i}}\left(\mathbf{x}_{2}\right)\right)\right] \\
& =\frac{1}{p} \ln \sum_{i=1}^{m}\left\{\exp \left[p\left(-\mu_{C_{i}}\left(\mathbf{x}_{1}\right)\right)\right]\right\}^{\lambda}\left\{\exp \left[p\left(-\mu_{C_{i}}\left(\mathbf{x}_{2}\right)\right)\right]\right\}^{1-\lambda}
\end{aligned}
$$

The Hölder inequality [9] implies that

$$
\begin{aligned}
\sum_{i=1}^{m}\left\{\exp \left[p\left(-\mu_{C_{i}}\left(\mathbf{x}_{1}\right)\right)\right]\right\}^{\lambda}\left\{\exp \left[p\left(-\mu_{C_{i}}\left(\mathbf{x}_{2}\right)\right)\right]\right\}^{1-\lambda} \\
\leq\left\{\sum_{i=1}^{m} \exp \left[p\left(-\mu_{C_{i}}\left(\mathbf{x}_{1}\right)\right)\right]\right\}^{\lambda}\left\{\sum_{i=1}^{m} \exp \left[p\left(-\mu_{C_{i}}\left(\mathbf{x}_{2}\right)\right)\right]\right\}^{1-\lambda} .
\end{aligned}
$$

Consequently,

$$
\begin{aligned}
F_{p}\left(\lambda \mathbf{x}_{1}+(1-\lambda) \mathbf{x}_{2}\right) & \leq \frac{\lambda}{p} \ln \sum_{i=1}^{m} \exp \left[p\left(-\mu_{C_{i}}\left(\mathbf{x}_{1}\right)\right)\right]+\frac{1-\lambda}{p} \ln \sum_{i=1}^{m} \exp \left[p\left(-\mu_{C_{i}}\left(\mathbf{x}_{2}\right)\right)\right] \\
& =\lambda F_{p}\left(\mathbf{x}_{1}\right)+(1-\lambda) F_{p}\left(\mathbf{x}_{2}\right) .
\end{aligned}
$$

This shows that $F_{p}(x)$ is a convex function on $\mathbb{R}^{n}$.

Lemma 2.1 directly leads to the following result.

THEOREM 2.2. For the system of fuzzy linear inequalities (1.1), if $\mu_{C_{i}}(\mathbf{x}), i=1,2, \ldots$, $m$, are concave, then we can find a solution to (1.1) by solving the unconstrained convex programming problem (2.12) with a sufficiently large $p$.

3. A numerical example. Consider the following system of fuzzy linear inequalities [10]:

$$
\begin{aligned}
& f_{1}(\mathbf{x})=-4 x_{1}-5 x_{2}-9 x_{3}-11 x_{4}+111.57 \leqq 0, \\
& f_{2}(\mathbf{x})=x_{1}+x_{2}+x_{3}+x_{4}-15 \leqq 0, \\
& f_{3}(\mathbf{x})=7 x_{1}+5 x_{2}+3 x_{3}+2 x_{4}-80 \leqq 0, \\
& f_{4}(\mathbf{x})=3 x_{1}+5 x_{2}+10 x_{3}+15 x_{4}-100 \leqq 0,
\end{aligned}
$$


where the membership function $\mu_{C_{i}}(\mathbf{x}), i=1,2,3,4$, are specified as follows:

$$
\begin{aligned}
& \mu_{C_{1}}(\mathbf{x})= \begin{cases}1, & \text { if } f_{1}(\mathbf{x}) \leq 0, \\
1-\left(\frac{f_{1}(\mathbf{x})}{10}\right)^{2}, & \text { if } 0<f_{1}(\mathbf{x}) \leq 10, \\
0, & \text { if } f_{1}(\mathbf{x})>10,\end{cases} \\
& \mu_{C_{2}}(\mathbf{x})= \begin{cases}1, & \text { if } f_{2}(\mathbf{x}) \leq 0, \\
1-\left(\frac{f_{2}(\mathbf{x})}{5}\right)^{3}, & \text { if } 0<f_{2}(\mathbf{x}) \leq 5, \\
0, & \text { if } f_{2}(\mathbf{x})>5,\end{cases} \\
& \mu_{C_{3}}(\mathbf{x})= \begin{cases}1, & \text { if } f_{3}(\mathbf{x}) \leq 0, \\
1-\left(\frac{f_{3}(\mathbf{x})}{40}\right)^{2}, & \text { if } 0<f_{3}(\mathbf{x}) \leq 40, \\
0, & \text { if } f_{3}(\mathbf{x})>40,\end{cases} \\
& \mu_{C_{4}}(\mathbf{x})= \begin{cases}1, & \text { if } f_{4}(\mathbf{x}) \leq 0, \\
1-\left(\frac{f_{4}(\mathbf{x})}{30}\right)^{2}, & \text { if } 0<f_{4}(\mathbf{x}) \leq 30, \\
0, & \text { if } f_{4}(\mathbf{x})>30 .\end{cases}
\end{aligned}
$$

Applying Bellman and Zadeh's method of fuzzy decision making [1], the maximizing solution $\mathbf{x}^{*}$ of this problem is given by solving the problem

$$
\max _{\mathbf{x} \in \mathbb{R}^{n}} \min \left\{1-\left(\frac{f_{1}(\mathbf{x})}{10}\right)^{2}, 1-\left(\frac{f_{2}(\mathbf{x})}{5}\right)^{3}, 1-\left(\frac{f_{3}(\mathbf{x})}{40}\right)^{2}, 1-\left(\frac{f_{4}(\mathbf{x})}{30}\right)^{2}\right\},
$$

which is equivalent to the min-max problem

$$
-\min _{\mathbf{x} \in \mathbb{R}^{n}} \max \left\{\left(\frac{f_{1}(\mathbf{x})}{10}\right)^{2}-1,\left(\frac{f_{2}(\mathbf{x})}{5}\right)^{3}-1,\left(\frac{f_{3}(\mathbf{x})}{40}\right)^{2}-1,\left(\frac{f_{4}(\mathbf{x})}{30}\right)^{2}-1\right\} .
$$

An $\epsilon$-optimal solution of the min-max problem can be obtained by solving the unconstrained convex programming problem

$$
\begin{gathered}
-\min _{\mathbf{x} \in R^{n}} \frac{1}{p} \ln \left\{\exp \left[p\left(\left(\frac{f_{1}(\mathbf{x})}{10}\right)^{2}-1\right)\right]+\exp \left[p\left(\left(\frac{f_{2}(\mathbf{x})}{5}\right)^{3}-1\right)\right]\right. \\
\left.+\exp \left[p\left(\left(\frac{f_{3}(\mathbf{x})}{40}\right)^{2}-1\right)\right]+\exp \left[p\left(\left(\frac{f_{4}(\mathbf{x})}{30}\right)^{2}-1\right)\right]\right\}
\end{gathered}
$$

with $p$ being sufficiently large.

Taking $p=100$, the algorithm terminated at the point $\mathbf{x}^{*}=(15.40,-15.16,18.44$, $-3.65)^{T}$. 
4. Concluding remarks. In this paper, a system of fuzzy linear inequalities is studied. It shows that solving such problems can be reduced to a regular min-max problem. An entropic regularization method is introduced for solving the resulting problem. This method essentially provides a smooth and uniform approximation for solving the min-max problem. Only a commonly used BFGS subroutine is required in our implementation. The required software development effort is minimal.

Acknowledgment. The author would like to thank professor Shu-Cherng Fang for his very constructive and valuable suggestions.

\section{REFERENCES}

[1] R. E. Bellman and L. A. Zadeh, Decision-making in a fuzzy environment, Management Sci. 17 (1970/1971), B141-B164.

[2] D. P. Bertsekas, Constrained Optimization and Lagrange Multiplier Methods, Computer Science and Applied Mathematics, Academic Press, New York, 1982.

[3] C. Charalambous and A. R. Conn, An efficient method to solve the minimax problem directly, SIAM J. Numer. Anal. 15 (1978), no. 1, 162-187.

[4] G. Di Pillo, L. Grippo, and S. Lucidi, A smooth method for the finite minimax problem, Math. Programming, Ser. A 60 (1993), no. 2, 187-214.

[5] C. Gígola and S. Gómez, A regularization method for solving the finite convex min-max problem, SIAM J. Numer. Anal. 27 (1990), no. 6, 1621-1634.

[6] J.-B. Hiriart-Urruty and C. Lemaréchal, Convex Analysis and Minimization Algorithms. I, Grundlehren der Mathematischen Wissenschaften, vol. 305, Springer-Verlag, Berlin, 1993.

[7] _ Convex Analysis and Minimization Algorithms. II, Grundlehren der Mathematischen Wissenschaften, vol. 306, Springer-Verlag, Berlin, 1993.

[8] E. T. Jaynes, Information theory and statistical mechanics, Phys. Rev. (2) 106 (1957), no. 4, 620-630.

[9] N. D. Kazarinoff, Analytic Inequalities, Holt, Rinehart and Winston, New York, 1961.

[10] Y.-J. Lai and C.-L. Hwang, Fuzzy Mathematical Programming. Methods and Applications, Lecture Notes in Economics and Mathematical Systems, vol. 394, Springer-Verlag, Berlin, 1992.

[11] X.-S. Li and S.-C. Fang, On the entropic regularization method for solving min-max problems with applications, Math. Methods Oper. Res. 46 (1997), no. 1, 119-130.

[12] D. G. Luenberger, Linear and Nonlinear Programming, Addison-Wesley, Massachusetts, 1984.

[13] E. Polak, D. Q. Mayne, and J. E. Higgins, Superlinearly convergent algorithm for min-max problems, J. Optim. Theory Appl. 69 (1991), no. 3, 407-439.

[14] R. A. Polyak, Smooth optimization methods for minimax problems, SIAM J. Control Optim. 26 (1988), no. 6, 1274-1286.

[15] A. Vardi, New minimax algorithm, J. Optim. Theory Appl. 75 (1992), no. 3, 613-634.

[16] I. Zang, A smoothing-out technique for min-max optimization, Math. Programming 19 (1980), no. 1, 61-77.

[17] H.-J. Zimmermann, Fuzzy Set Theory - and Its Applications, Kluwer Academic Publishers, Massachusetts, 1992.

F. B. LiU: DePartment of MechanicAl ENGINEERING, I-SHOU UNIVERSity, KAOHSIUNG 840, TAIWAN

E-mail address: f1iu@isu.edu.tw 


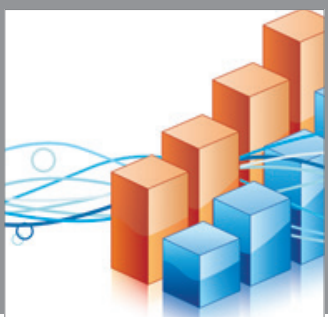

Advances in

Operations Research

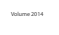

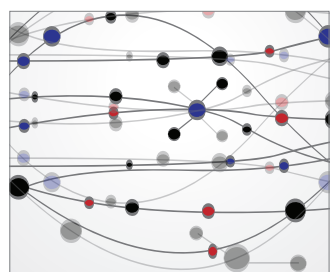

\section{The Scientific} World Journal
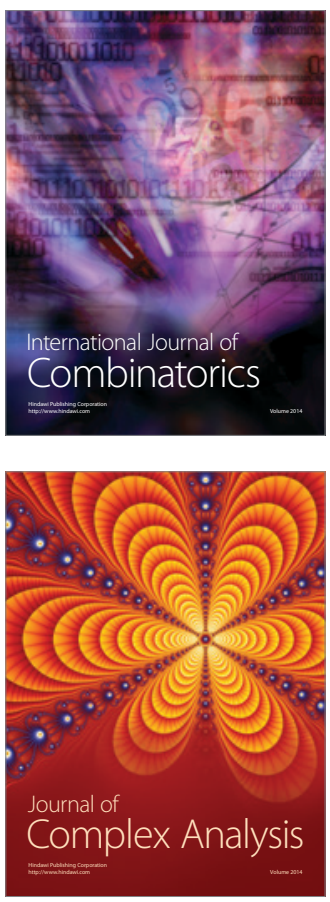

International Journal of

Mathematics and

Mathematical

Sciences
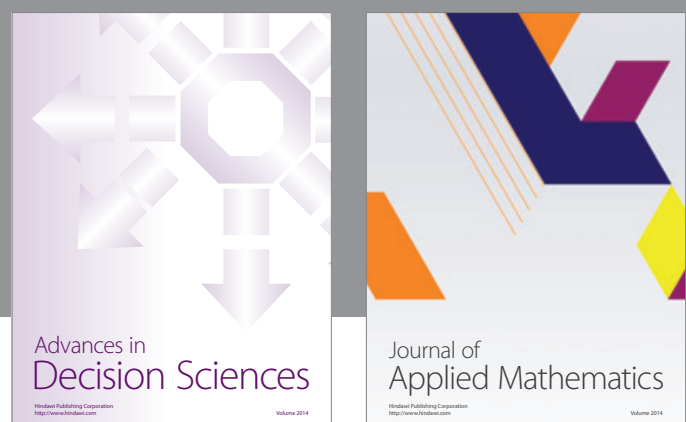

Journal of

Applied Mathematics
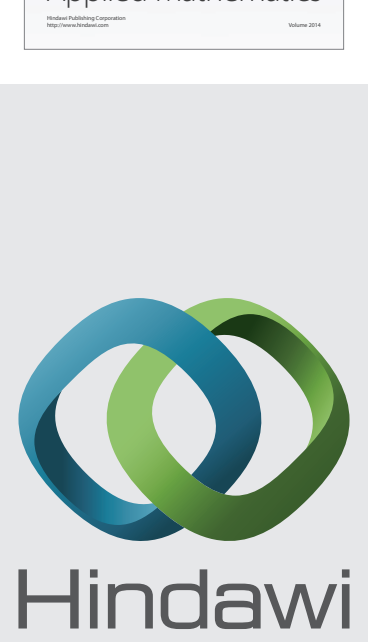

Submit your manuscripts at http://www.hindawi.com
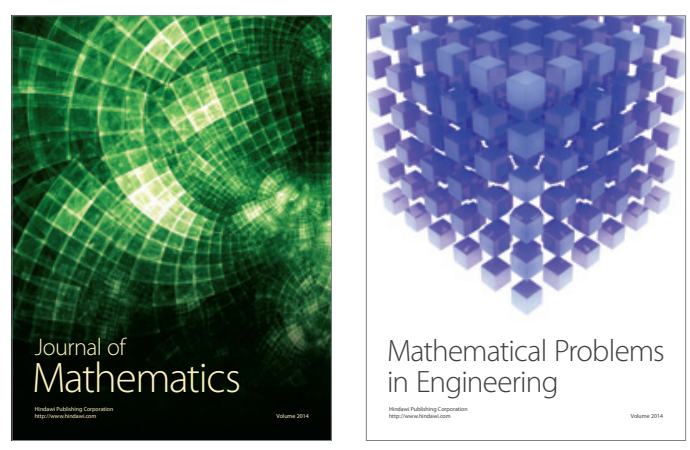

Mathematical Problems in Engineering
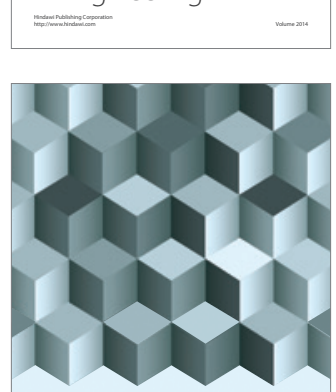

Journal of

Function Spaces
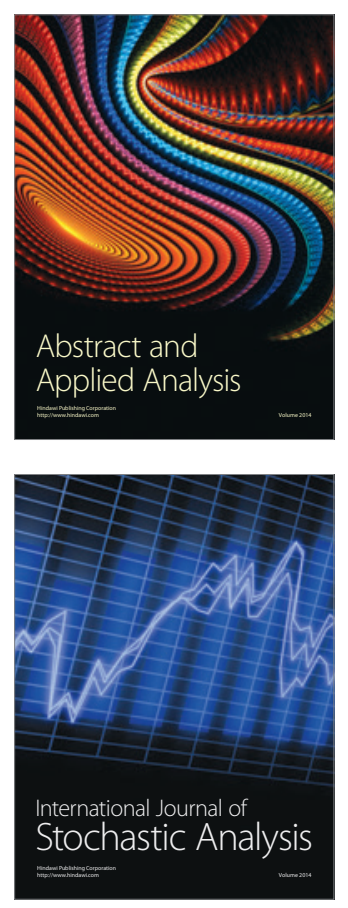

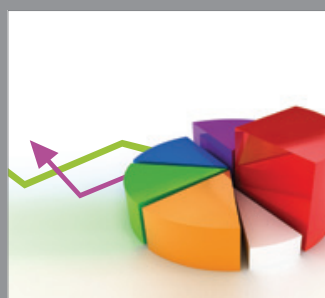

ournal of

Probability and Statistics

Promensencen
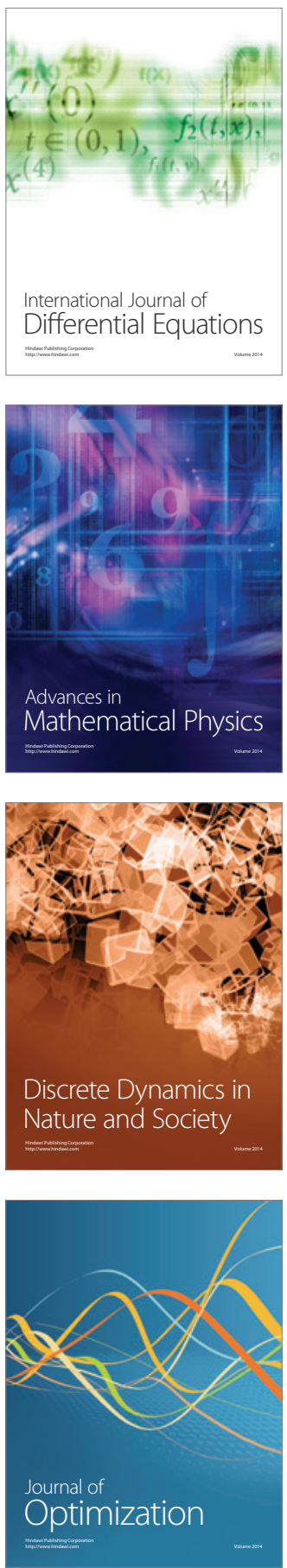